\title{
Cooking with the Elements: Intuitive Immersive Interfaces for Augmented Reality Environments
}

\author{
Leonardo Bonanni, Chia-Hsun Lee, and Ted Selker \\ MIT Media Laboratory, \\ 20 Ames Street, Cambridge MA 02139 (USA) \\ \{amerigo, jackylee, selker\}@media.mit.edu \\ http://www.media.mit.edu/ amerigo
}

\begin{abstract}
The glut of information produced by ubiquitous computing in augmented reality environments requires that the resulting information displays be tailored to the attention of users and mapped directly to the objects and surfaces of the space. This paper proposes a method for designing and implementing ambient information displays combining ambient displays and augmented reality to produce useful intuitive interfaces that are concretely mapped to architectural spaces for the purposes of expanding and enriching the quality and sensuality of user experience.
\end{abstract}

\section{Introduction}

Ubiquitous computing in architectural space is making it possible to gather information about people and their tasks that can be applied to augmenting and enriching the experience of users. As the interface possibilities made possible by the widespread use of computer sensors and effectors grow, it becomes possible to map information on all the surfaces and tools of a space to orient and assist users and make possible entirely new experiences within conventional environments. In order to be effective these spatial interfaces must be designed and implemented in accordance with the attention of users and their performance so as to assist and enrich user experience without distracting or confusing basic tasks. Ambient interfaces offer a minimally

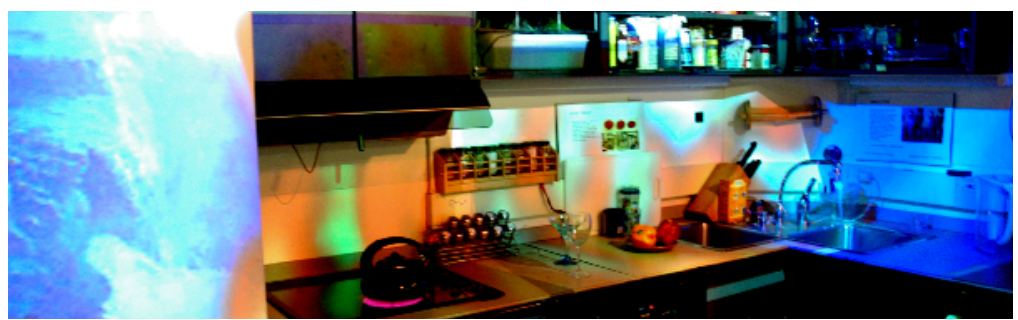

Fig. 1. Cooking with the Elements: Multimedia projections enrich a conventional kitchen by projecting intuitive displays to reveal the status of tools and surfaces 
taxing means of distributed information display, while augmented reality interfaces seek to provide useful task-based information. This paper proposes a new type of interface that concentrates on creating intuitive immersive interfaces that inform activity without interfering with tasks.

\section{Related Work}

Ambient displays have been prototyped and produced for the purpose of displaying information throughout the built environment in a manner that does not tax the attention of users. Ishii's Windmills and Ambient Devices' Stock Orb are two systems that display a limited amount of information in the form of decorative objects $[1,2]$. These devices purportedly communicate simple information in a language only understood by their trained owners; for example, the Orb requires users to know that its color refers to stock market values and that red indicates decreasing market performance while yellow indicates no change. Similarly, Kaye's InStink utilizes computerdispensed perfumes as an ambient interface that maps information to the more salient but still unintuitive olfactory sense [4]. Nevertheless, ambient interfaces have the benefits of using the minimum possible attentional "bandwidth" to communicate information and organizing the information by distributing it throughout a physical space.

At the other extreme, spatial information displays have been implemented to orient and assist users in tasks by presenting, for example, projected desktop instruments in an office [6] or recipes in a kitchen [3]. In our evaluations of such text- and graphicheavy displays, it was determine that they had no benefit and in some cases performed worse than standard screen- or paper-based displays. Reasons include the cognitive weight of text and graphics, especially when distributed over large areas, and the inability to reference multiple sources of information simultaneously. However these displays have the distinct advantage of mapping digital information directly to the physical space where tasks are being performed; for example, in Microsoft's Kitchen of the Future users measure out flour by pouring it onto a projected circle until it is completely covered [4].

\section{Implementation}

Our interfaces seek to combine the attention-based design of ambient interfaces with the utility of one-to-one projected augmentation by overlaying an environment with intuitive, immersive information interfaces. Our test bed is a kitchen because it stands as the epitome of a feedback-less modern space. Centuries of innovation in hygiene and automation have eliminated most sensory experience from modern kitchens while filling them with ubiquitous computers, sensors and effectors. In our kitchen, we have installed a system of sensors and projectors specifically for the purpose of overlaying the existing space with sensory feedback to assist and enrich the user's experience. The research on which this paper is based originates with a case study that considers the faucet alone, and expands to consider the appliances and countertops of the space. 


\subsection{HeatSink}

In the kitchen, users have come to depend on remote controls and indicators to know the temperature of food and water or the status of the stove. For example, how often do we scald ourselves at the faucet or wait arbitrarily for tap water to reach a desired temperature? HeatSink is a simple solid-state circuit that projects colored light into the stream of tap water to indicate its temperature intuitively: red for hot fading to blue for cold. During design one iteration was considered that maps temperature to a full red-green-blue spectrum like the Stock Orb. The final choice of simple red and blue was based on the fact that people do not intuitively understand the temperature of 'green' water; their main concern is to determine whether the water is colder or hotter than their hands before touching it. By using various intensities of only two colors, HeatSink displays only the minimum essential information and does not inconvenience the task at hand or require prior knowledge. Taking a cue from projected augmented reality interfaces, the projection of colored light directly into the stream proves more successful than remote indicators like the control knob because the information is overlaid directly on the user's focus of attention (see Fig. 2).

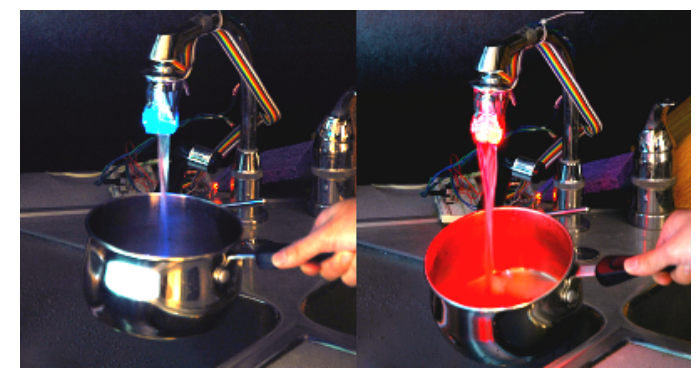

Fig. 2. HeatSink: LEDs mounted to the faucet aerator and driven by a PIC-based microcircuit project modulated colored light into the stream of tap water

\subsection{Cooking with the Elements}

The modern kitchen is a technological marvel that combines the elements of fire, water, ice and earth in a compact hygienic space. Following from HeatSink, Cooking with the Elements maps intuitive multimedia textures to the countertops of a conventional kitchen to enrich and inform tasks in the space. Common problems such as knowing if the oven is hot or keeping the refrigerator door open too long can be intuitively annotated with dynamic audiovisual textures projected onto the surfaces of the appliances themselves. Likewise, the countertop can serve as a control panel that communicates the status of tools and surfaces intuitively in an ambient way that responds to the attention of users according to their performance and position in space.

Cooking with the elements consists of tiled multimedia projections that seamlessly cover all the countertops of the kitchen. Proximity sensors situated along the countertop edge locate users while temperature and water sensors and micro-switches detect the status of the cabinets, countertops, sink, and appliances. A Director movie is generated across three seamlessly tiled projections that maps dynamic multimedia 
textures to the space depending on the status of tools and the performance of users. When someone opens the refrigerator, the sound of a cold wind plays and projected snow begins to accumulate as an indication of how long the door is open and the energy wasted. When the electric range is on or the stove reaches desired temperature, a dynamic fire is projected while the crackling of a wood fire is heard. If the sink is left running, a projected pool of water grows to cover the countertop while the sound of a creek fills the room. Depending on where users are located, these displays grow or shrink to remain in the periphery of their attention and never to detract from their current task. In case a user forgets the water running or the stove on, the displays grow so that anyone entering the space is immediately aware that something is wrong. Although the displayed textures only convey limited information (hot, cold, wet) they seek to do so in a completely intuitive manner that is always accessible and never annoying. Cooking with the Elements enriches the sensory nature of cooking and returns some of the feedback that was lost when kitchens became modern and hermetic (see Fig. 1).

\section{Conclusion}

As computers become ubiquitous in the built environment, interfaces that interact at the scale of architectural space will be commonplace. Rather than fill the space with text and graphics, these immersive multimedia displays have the power to transform and augment human experience so that mundane experiences become exciting and users understand information with minimal cognitive effort or prior knowledge. While screen- and paper-based devices will continue to provide us with highly informative interfaces, the space around us can become simpler and more beautiful with immersive interfaces. Imagine waking up to a projected sunrise rather than a beeping alarm or smelling fresh food from the market when you open the refrigerator - computer interfaces can actively enrich and augment our daily experiences by calmly and intuitively enhancing the bland textures of our built environment with dynamic sensory experiences as we once had and as we never before dreamed.

\section{References}

1. Ambient Devices: www.ambientdevices.com

2. Ishii, H., Ren, S. and Frei, P., Pinwheels: Visualizing Information Flow in an Architectural Space (short paper), in Proc. CHI' $01,111-12$.

3. Ju, W. et. al. (2001). "Counteractive: An Interactive Cookbook for the Kitchen Counter," in Extended Abstracts CHI 2001, 269-70.

4. Kaye, J. N. (2001) Symbolic Olfactory Display. Master's Thesis, MIT Media Lab, 2001.

5. Microsoft Kitchen of the Future as seen in the Food Network's documentary 'Kitchens of the Future,' 2003.

6. Wellner, P. "The DigitalDesk calculator: Tangible Manipulation on a Desk Top Display," in Proc. UIST' '91, 27-34. 